\title{
Software Defined Networking for Energy Harvesting Internet of Things
}

\author{
Xumin Huang, Rong Yu, Member, IEEE, Jiawen Kang, Zhuoqun Xia and Yan Zhang, Senior Member, IEEE
}

\begin{abstract}
Internet of things (IoT) provides ubiquitous intelligence and pervasive interconnections to diverse physical objects. The overall network performance of existing IoT is restricted by limited network lifetime. Hence, energy harvesting technology with energy replenishment from mobile charger is proposed to prolong the network lifetime. Energy harvesting IoT is emerged. Nodes can not only request energy replenishment from the mobile charger, but also transfer surplus energy to the mobile charger for improving energy utilization. This gives rise to bi-directional energy flows in the network. A new paradigm that energy flows coexist with data flows is further resulted in. But there exist great challenges on controlling these flows. Toward centralized flow control, we exploit software defined networking to simplify and optimize network management, thus introduce software defined energy harvesting Internet of things (SEANET). In our proposed architecture, the data plane, energy plane and control plane are decoupled to support enhanced communications and flexible energy scheduling. We consider reliable communications for SEANET, and propose to relay data packets among the nodes with high reputation values and sufficient energy. In particular, reputation values of nodes are computed by the multi-weighted subjective logic for higher accuracy. Besides, a Nash bargaining game is formulated to solve the benefit allocation problem for energy trading in SEANET. Numerical results indicate that SEANET improves data traffic by reducing packet loss, optimizes energy utilization and saves energy.
\end{abstract}

\section{INTRODUCTION}

Internet of Things (IoT) as an emerging paradigm provides ubiquitous intelligence and pervasive interconnections to a vast number of diverse physical objects in our daily life. Enabled by advanced technologies, e.g., RFID and communication technologies, the physical objects can not only "see", "hear" and "talk" with each other but also "think" independently [1]. They communicate and cooperate with each other for

This work was supported in part by programs of NSFC under Grant nos. $61379115,61422201,61501127,61370159$ and U1301255, U1501251, the Science and Technology Program of Guangdong Province under Grant no. 2015B010129001, 2015B010106010, 2016A030313705, 2014B090907010, 2015B010131014, Special-Support Project of Guangdong Province under grant no. 2014TQ01X100, Science and Technology Program of Guangzhou under grant no. 2014J2200097, and is partially supported by the projects 240079/F20 funded by the Research Council of Norway. (Corresponding author: Yan Zhang.)

Xumin Huang, Rong Yu, and Jiawen Kang are with School of Automation, Guangdong University of Technology, and also with Guangdong Key Laboratory of IoT Information Technology, Guangzhou 510006, China. Email: huangxu_min@163.com, yurong@ieee.org, kjwx886@163.com.

Zhuoqun Xia is with Hunan Provincial Key Laboratory of Intelligent Processing of Big Data on Transportation, Changsha University of Science and Technology, Changsha 410114, Hunan Province, China. Email: xiazhuoqun@csust.edu.cn.

Yan Zhang is with Department of Informatics, University of Oslo, Norway. Email: yanzhang@ieee.org. intelligent decision making. Owing to the intelligence, IoT applications have been widely deployed in household, industry and business areas [2]. Typical applications include in-home healthcare, environmental monitoring and social networking [3]. To support the applications, green energy supply and usage play an essential role in improving overall network performance. Energy harvesting technology has been proposed to achieve long-term and self-sustainable operations for nodes and base stations [4], [5]. Thus, nodes perform data communication and energy harvesting simultaneously. As a result, energy flows coexist with data flows in energy harvesting IoT.

Recently, mobile charger has been proposed to offer energy replenishment to nodes and further prolong network lifetime. Energy-deficient nodes can "download" energy from a mobile charger, establishing an energy link named energy downlink. In turn, once nodes stay idle and continuously harvest energy, they can accumulate and "upload" their surplus energy to the mobile charger, which forms an energy uplink. Hence, energy flows between the mobile charger and nodes are bidirectional. The bi-directional energy flows promote largescale energy scheduling because energy can be collected from, or transferred to nodes in different areas. This addresses the limitation caused by the distance among nodes in energy cooperation [6]. After forwarding the energy flows, energy states of nodes are updated. The control of the data flows takes the nodes' current energy states into consideration for reliable communications. With data transmission, the energy states of the nodes are changed and new energy flows among nodes will be formed on demand. Clearly, the data flows are strictly coupled with the energy flows.

This gives rise to critical challenges about optimal control of these two flows. A large amount of data traffic is generated by heterogeneous devices in existing IoT, so traffic control need to be highly scalable [7]. To efficiently utilize the bidirectional energy flows, energy scheduling is also required to be much more elastic. To summarize, simplified network management is necessary, and control logic of the two flows can be abstracted from underlying implementations. Moreover, for joint optimization of network management, a global view with logically centralized characteristic should be realized to make optimal decisions dynamically.

To satisfy the aforementioned requirements, we exploit the software defined networking (SDN) technology to provide intelligent network management for energy harvesting IoT, resulting in software defined energy harvesting Internet of things (SEANET). SDN decouples the control plane and data plane, and offers efficient network control for wired and wireless networks [8]. By extending programmability of 
network elements, nodes only perform flow forwarding tasks while decision-making capabilities are shifted to the centralized control plane. This is beneficial for simplifying policy enforcement, facilitating network configuration to deploy new features, and finally smoothing network evolution. Nowadays, SDN has been applied to IoT for performance improvement but mainly in terms of architecture presentation [9], protocol design [10] and security guarantee [11].

In this paper, we focus on efficient utilization of SDN for advanced IoT and propose SEANET. We present an enhanced SDN-based architecture wherein the data plane, energy plane and control plane are decoupled, aiming to jointly optimized control of the data flows and energy flows. In particular, the energy plane is abstracted as the bi-directional energy flows arise up and coexist with the data flows. By extending the programmability of these planes, SEANET brings unprecedented improvement to data communications, high flexibility to energy scheduling and optimizes energy utilization, consequently promoting network development. Unlike the work in [10] and [12], we consider both data communications and energy scheduling simultaneously for the SEANET. We propose reliable communications for nodes and formulate a feasible energy trading model between the mobile charger and the nodes to support large-scale energy scheduling.

We summarize main contributions of the paper as follows.

- We apply SDN to energy harvesting IoT and design the hardware structure and hierarchical architecture for SEANET, which offers centralized control logic for coexisted energy flows and data flows in IoT.

- For secure communications, we leverage the multiweighted subject logic to evaluate reputation values of nodes with higher accuracy. Data packets are relayed among the nodes with remarkable reputation values and sufficient energy for reducing packet loss.

- Toward flexible energy scheduling, a Nash bargaining game is formulated to solve the benefit allocation problem in the energy trading. Thus, energy-rich nodes make optimal decisions to upload the surplus energy to the mobile charger. We also justify that the game can reach the Nash equilibrium finally.

The rest of the paper is organized as follows. Section II presents the related work. We introduce the proposed SEANET with details in Section III. In Section IV, we consider both the reputation values and energy states of nodes for relay selection when routing traffic in SEANET. The energy trading problem is formulated and solved by using the Nash bargaining game in Section V. We present numerical results about performance evaluation in Section VI and draw conclusions in Section VII.

\section{RELATED WORK}

In recent years, SDN technology has drawn great attention in IoT because of the high programmability to network elements and flexible managing capabilities for network optimization. The authors in [7] studied the opportunities and challenges of adopting SDN to promote the development of next generation IoT applications. The work introduced several user cases wherein SDN is used for home networking, mobile applications, online multimedia services and so on. Toward rapid evolution of IoT, an SDN-based architecture coupled with network function virtualization is presented to cope with different challenges in IoT environment [9]. In this SDN-based architecture, the interoperability of IoT devices is solved. The software-centric management also avoids hardware limitations, making service chain shorter and simpler.

SDN is integrated into IoT for great improvements but this also gives rise to many new problems. Security issues are inevitable due to the vulnerabilities of communication channels between SDN controllers and SDN switches. The work in [11] considered potential threats of man-in-the-middle attacks on the OpenFlow control channels. To detect the attack, the authors proposed a countermeasure using Bloom filter. At the same time, traffic routing and energy usage can also be optimized, owing to the proposal of SDN in IoT. In [10], the authors presented a multi-hop routing protocol by using the centralized control from an SDN controller. The location, neighbors and energy status are obtained and updated for providing the shortest path and disjoint multipath routing in the network. By efficiently programming, a sensor node in software-defined sensor networks is capable of conducting multiple sensing tasks simultaneously [12]. But this results in a problem that how to design an optimal management strategy of the nodes for ensuring quality-of-sensing for all tasks. To solve the problem, a mixed-integer with quadratic constraints programming problem is formulated and the authors finally developed an efficient online algorithm to solve it.

Compared with the existing work, our work pay attention to exploit SDN for improving network management of energy harvesting IoT. The advanced IoT is emerged when energy harvesting technology and the mobile charger are combined and cooperate well to prolong network lifetime. Bi-directional energy flows are comprised of energy donwlinks and energy uplinks between nodes and the mobile charger. Dynamic energy flows coexist with diverse data flows and this results in a core problem about the optimal control for these two flows. The challenge that energy flows couple with data flows is neglected by most of existing work, e.g., [10] and [12]. To cope with the problem, we abstract the energy plane and separate the control plane from the data plane and energy plane. Control logic is split from underlying implementations, and becomes centralized by obtaining network states in real time. The network management is improved finally.

\section{Software Defined EnERGy Harvesting IoT}

\section{A. Hardware Structure}

For a node, it is equipped with a sensor, an actuator, a data buffer, an antenna and a battery. Nodes transmit and receive sensory data generated in monitoring areas, resulting in changes of the data queue. Data transmission clearly leads to energy consumption. With energy harvesting devices, nodes harvest renewable energy (e.g, solar and wind) from ambient environment. The renewable energy makes a positive contribution to the energy queue in the battery. The node can also request energy replenishment from a mobile charger acting as an energy transporter. Meanwhile, the mobile charger collects surplus energy from energy-rich nodes. 


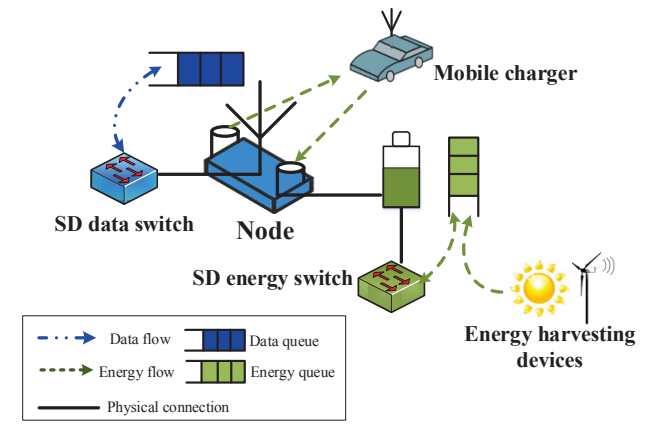

Fig. 1: Hardware structure for nodes in SEANET .

To enable the bi-directional energy transfer, there are an oscillator, an AC/DC converter, two resonant coils including transmitting/receiving coils in the mobile charger and nodes to support bidirectional energy transfer. Here, energy transfer from point to point (i.e., non-radiative energy transfer) is attained by establishing an oscillating magnetic field between the transmitting coil and the receiving coil. When the mobile charger charges a node via an energy downlink, the oscillator converts DC from its battery into high-frequency AC. The transmitting coil in the mobile charger works as an energy transmitter. By establishing an oscillating magnetic field between the transmitting coil and the receiving coil, an $\mathrm{AC}$ is then induced in the receiving coil and is regulated to charge the battery of the node quickly [13]. Reverse operations are adjusted by the node and mobile charger to support an energy uplink. More details can be found in our work [14].

As shown in Fig. 1, the hardware structure is upgraded to realize SEANET. SDN switches are newly added to connect with nodes. In SEANET, there exist two kinds of software-defined (SD) switches: SD data switches and SD energy switches. They are physically connected to antenna and battery, respectively. So the SD switch status is directly related to data queue and energy queue of the nodes. The SD data switches forward data flows among the nodes while the SD energy switches forward energy flows between the mobile charger and nodes. Thus, data transmission and energy transfer are managed well by controlling the SDN switches efficiently.

\section{B. Hierarchical Architecture}

SEANET offers excellent programmability to data transmission and energy transfer, and adapts to various applications via efficient programming interfaces. The essential feature of SEANET is to logically separate the control plane, data plane and energy plane. The energy plane and data plane are to perform energy scheduling and data transmission tasks, respectively. The centralized control plane obtains network states to optimize flow forwarding dynamically. The flow rules are totally implemented by the software defined data controllers: SD data controller and SD energy controller respectively in the control plane. The rules are sent to the data plane and energy plane to instruct the SD data switches and SD energy switches. Followed by the instructions, the nodes take actions on incoming data packets and energy units. To realize the separation of the planes in SEANET, a well-defined API

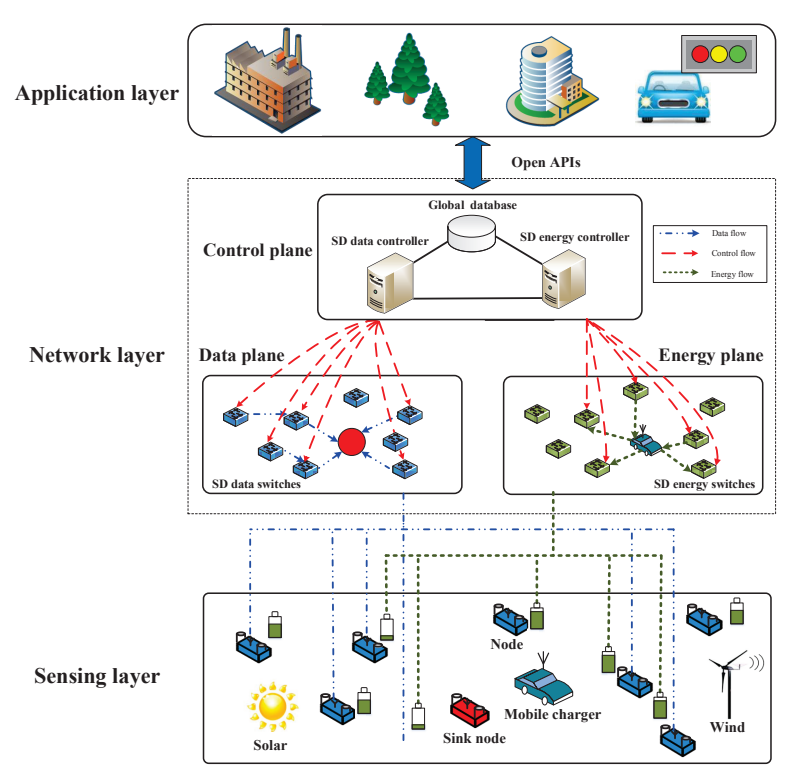

Fig. 2: Hierarchical architecture for SEANET

between the switches and the controllers is crucial. A typical example of such an API is OpenFlow protocol [15].

As shown in Fig. 2, the proposed SEANET architecture consists of three layers: sensing layer, network layer and application layer. In the bottom layer, nodes are of various functionalities, e.g., querying location, monitoring temperature and tracking motion. The network layer provides heterogeneous connectivity and undertakes regular network management tasks, e.g., traffic control, energy scheduling, security guarantee and fault handling. The key layer is divided into three separated planes. Nodes are abstracted as simple flow forwarding devices in the data plane and energy plane. All the decision-making capabilities are assigned to the control plane. As for the top layer, network administrators are convenient to make modifications, deploy new features and upgrade services according to application requirements. More details about the layers are given as follows.

1) Application layer: A variety of IoT applications, e.g, industrial wireless sensor networks, environmental monitoring, smart buildings and intelligent transportation systems, are easier to be authorized, supported and developed in the architecture. By using open APIs, external service providers are allowed to customize the behavior of the network according to different requirements. With consideration of the specifics of each application, high-level network policies are created well without any hardware changes.

2) Network layer: The network layer consists of vital SDN components: SD switches and SD controllers. The switches forward the data flows and energy flows based on the flows instead of destinations and the controllers make optimal forwarding decisions with global optimum.

Control Plane: There exist two programmable logical controllers and a global database. The database stores monitoring information of network states, e.g., network traffic and energy harvesting characteristics of the nodes. By analyzing the real-time monitoring information, the SD data controller and 
SD energy controller dynamically schedule the data flows and energy flows. Two SDN controllers communicate and cooperate with each other to ensure logically centralized control. The controllers also acquire status information from the switches for necessary fault handling. To sum up, the controllers establish secure connections with the switches, read their current states to check whether at work, and install control instructions in them.

Data Plane: The SD data switches execute the control instructions to forward sensory data among nodes. Data packets from different devices are directed along the optimal paths by considering heterogeneity of devices, network topology, energy states of nodes and so on. In particular, data packets are relayed among those nodes with cooperative behavior and sufficient energy for reliable communications. This aims to reduce packet loss and greatly improves network throughput.

Energy Plane: The SD energy switches forward the bidirectional energy flows, and energy in the nodes can be scheduled elastically. In the energy plane, energy in the nodes' battery can be timely replenished by and upload to the mobile charger. The specified directions of the energy flows and the amount of replenished/uploading energy are strictly followed the instructions from the SD energy controllers. More nodes are benefited by the large-scale energy scheduling.

3) Sensing layer: In the sensing layer, the nodes involving tags, devices, sensors and equipments are able to automatically sense and intelligently identify things of ambient environment. The sensory data is generally transmitted to a sink node in the local monitoring areas via multiple-hop routing. To gather the sensory data, a mobile data collector is required in the network. Some existing work has proposed that the mobile charger can also act as the data collector to periodically travels around all the sink nodes to collect sensory data [16].

\section{Functional Block Diagram}

Fig. 3 shows the functional blocks in the SEANET, consisting of abstracted control logic and underlying physical infrastructures. With the surveillance of the entire SEANET, intelligent network control is achieved by the SD controllers via efficient coordinations. The nodes connecting with the switches act as simple flow forwarding devices, just perform certain operations followed by the control instructions. The functional blocks of SEANET are described as follows.

- Physical infrastructure: There are different physical entities, including a mobile charger, nodes with a battery, an antenna, and various energy harvesting devices. These physical entities are connected well to work as key enabling hardware for data transmission and energy transfer in SEANET. Especially, as the control logic is abstracted from underlying implementations, the physical infrastructures can be modified and upgraded independently.

- Network monitor: Through detecting events, monitoring information is comprised of two aspects: data and energy. Sensory data generation, link states and relay selection during traffic routing are known. Besides, the monitor supervises energy states of nodes, energy harvesting characteristics and bi-directional energy transfer process.

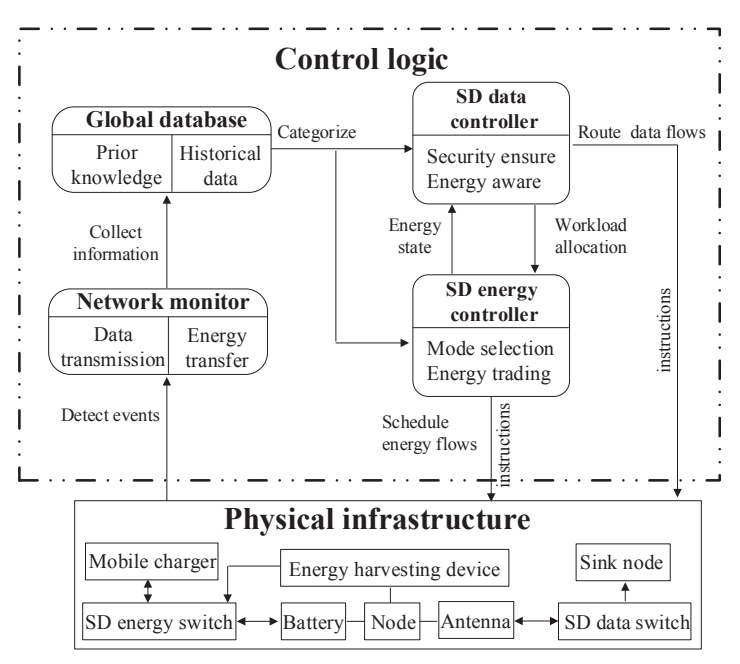

Fig. 3: Functional block diagram for SEANET.

- Global database: The monitoring information of network states is collected and stored in the global database. The historical interactions among any physical entities are also recorded for subsequently tracking.

- SD data controller: Based on prior knowledge, the SD data controller optimizes data traffic with consideration of security and efficiency. Toward secure communications, data packets are relayed among cooperative nodes. Trustworthiness evaluation is conducted to measure reputation of nodes. The controller also determines the optimal routing path according to energy states of nodes for reducing energy consumption and avoiding route interruption.

- SD energy controller: The SD energy controller firstly makes decisions for nodes about selection of energy transfer model. With different workloads, a node can work in an energy downlink or energy uplink mode. When the node becomes an energy-rich node, it can upload their surplus energy to the mobile charger. But this exists a benefit allocation problem between the node and the mobile charger from an economic perspective. So the SD energy controller also optimizes benefit allocation among them to implement energy trading well.

\section{Reliable data COMmUnications FOR SEANET}

\section{A. Reputation Value Formation in Data Communications}

Nodes relay data packets to their cooperative neighbors with high reputation for secure communications. In previous work, reputation is an essential metric to evaluate trustworthiness of nodes. Generally, misbehaving nodes act selfishly, deceitfully and even have malicious behaviours, e.g., dropping relaying data packets. As a consequence, these nodes have low reputation values. Each node prefers to interact with the higherreputation nodes to avoid potential security threats.

After monitoring behavior of neighbors, a node evaluates neighbor nodes' direct reputation values. In this paper, we utilize subjective logic to formulate the individual evaluation according to interaction histories among nodes. Subjective logic is a framework for probabilistic information fusion, which 
operates on subjective beliefs about the world [17]. Subjective logic uses the term "opinion" to denote the representation of a subjective belief, and models positive, negative statements and uncertainty.

We take node $i$ evaluates a reputation value for node $j$ as an example. After the interactions, the "opinion", that node $i$ holds for node $j$, is denoted as $w_{i: j}=\left(b_{i: j}, d_{i: j}, u_{i: j}, a_{i: j}\right)$. Here, $b_{i: j}$ and $d_{i: j}$ refer to the belief and disbelief of node $i$ in node $j$, respectively. $u_{i: j}$ is the uncertainty of node $i$ on node $j$ and $a_{i: j}$ is base rate. According to the work in [17], belief is the probability that the statement is true, disbelief is the probability that the statement is false, and uncertainty is the individual confidence in a node's knowledge on other nodes. The base rate is a predefined constant formed from an existing impression without solid evidences, which represents the willingness to believe other nodes. Generally, the base rate is set as 0.5 by default, similarly to the work [18] and [19]. Especially, the values of $b_{i: j}, d_{i: j}, u_{i: j}$ and $a_{i: j}$ and relationships among them are required by

$$
\begin{aligned}
& b_{i: j}, d_{i: j}, u_{i: j}, a_{i: j} \in[0,1] \\
& b_{i: j}+d_{i: j}+u_{i: j}=1 .
\end{aligned}
$$

In SEANET, the uncertainty $u_{i: j}$ in the reputation segment $w_{i: j}$ is mainly determined by the quality of link between node $i$ and node $j$. Here, the quality of link $q_{i, j}$ refers to packet success probability. To observe the behaviors of node $j, i n_{i, j}$ indicates the amount of received data packets from node $i$, while out $t_{i, j}$ is the amount of faithfully transmitted data packets relayed from node $i$. Based on $i n_{i, j}$ and $o u t_{i, j}$, the belief and disbelief values respectively are the percentage of transmitting and dropping data packets relayed from node $i$ within the scope of the confirmation, $1-u_{i: j}$.

$$
\begin{aligned}
& u_{i: j}=1-q_{i, j}, \\
& b_{i: j}=\left(1-u_{i: j}\right) \frac{\text { out }_{i, j}}{i n_{i, j}}, \\
& d_{i: j}=\left(1-u_{i: j}\right) \frac{i n_{i, j}-\text { out }_{i, j}}{\text { in }_{i, j}} .
\end{aligned}
$$

Using the above parameters, the reputation computation result $r_{i: j}=b_{i: j}+u_{i: j} a_{i: j}$ (i.e., reputation value) is to represent the expectation of node $i$ that node $j$ acts cooperatively.

\section{B. Multi-weighted Subjective Logic for Reputation Update in SEANET}

As nodes connect with the SD data switches, they upload their reputation segments to the SD data controller via communication protocols, e.g., OpenFlow. The SD data controller collects all the valid reputation segments about one targeted node and issues the reputation segments to the neighbor nodes of the target node. Then node $i$ can know one reputation segment from other neighbor nodes of node $j$, denoted as node $k(k \neq i)$. For node $i$, it also references the external reputation segments to update the latest reputation value of node $j$. But the reputation segments should be weighted properly for well utilization.

In the traditional subject logic, all the reputation segments are treated equally. But different reputation segments deriving from various providers should be weighted accordingly for being aggregated with improved accuracy. The providers always have distinct capabilities and conditions to observe the targeted node. For a reputation segment, if the rater has more prior knowledge on and updates more recent evaluation for the ratee, accuracy and availability of the reputation segment are much more remarkable. Besides, raters with similar observing conditions for the target node believe more in the reputation segments from each other.

Based on the vital principles, we propose the multi-weighted subjective logic for reputation update of nodes. More specifically, when the node $i$ figures out reputation value of the neighbor $j$, all the reputation segments (from node $k$ ) about the neighbor are collected and weighted adequately according to i) familiar degree of node $k$ for the target neighbor $j$, ii) context similarity between node $k$ and node $i$ when observing the target node $j$ and iii) updating time of the reputation segment from node $k$. In this way, node $i$ pays more attention to reputation segments with greater qualities and avoid the misdirection of coarse and unreliable reputation segments. As a result, highly accurate reputation update for neighbor $j$ is achieved finally. To sum up, the above three aspects of reputation segments are considered as follows:

Familiarity: For the reputation segment $w_{k: j}$, the familiarity value is to measure how much node $k$ (rater) is familiar with node $j$ (ratee). The higher familiarity value means that the rater has more prior knowledge on the ratee. We set that the familiarity value is evaluated by the number of past interactions among two nodes (denoted as $p_{k, j}$ ). With more interactions, node $k$ has more experience of monitoring the behavior of node $j$. When there exist $M$ neighbors of node $j$, the familiarity value between node $k$ and node $j$ considered by node $i$ denoted as $f_{k, j}$ can be calculated as

$$
f_{k, j}^{i}=\frac{(M-1) p_{k, j}}{\sum_{l=1, l \neq i}^{M} p_{l, j}} .
$$

Similarity: The similarity value is to measure how much the raters (nodes $i$ and $k$ ) are similar when forming their reputation segments about a common ratee (node $j$ ). We regard quality of link as main context characteristic to formulate the similarity value. When node $k$ has higher context similarity with node $i$, the reputation segment of node $k$ is emphasized. The similarity value between node $i$ and node $k$ when observing the target node $j$ is obtained by

$$
s_{i, k}^{j}=\frac{1}{1+\left|q_{i, j}-q_{k, j}\right|} .
$$

Freshness: The freshness is defined to measure the timeliness of forming the reputation segment. In historical records, the up-to-date reputation segments will be put a higher weight. We set the freshness $m_{k, j}^{i}$ with power-law distribution:

$$
m_{k, j}^{i}=\alpha_{1}\left(t_{i, j}-t_{k, j}\right)^{-\alpha_{2}},
$$

where $\alpha_{1}$ and $\alpha_{2}$ are two predefined parameters to adjust the influence of timeliness. $t_{i, j}$ and $t_{k, j}$ are the forming time of the reputation segment $w_{i: j}$ and $w_{k: j}$, respectively.

By considering the familiarity, similarity and freshness, the overall weight of the reputation segment $w_{k: j}$ for node $i$ is:

$$
v_{k, j}^{i}=\beta_{1} f_{k, j}^{i}+\beta_{2} s_{i, k}^{j}+\beta_{3} m_{k, j}^{i},
$$


where $\beta_{1}, \beta_{2}$ and $\beta_{3}$ are three predefined weighting factors and $\beta_{1}+\beta_{2}+\beta_{3}=1$.

The reputation segments from other neighbor nodes of node $j$ are aggregated with weights to form a synthesis reputation segment for node $i$, represented by $w_{i: j}^{\text {syn }}$. We consider that the synthetic reputation segment is weighted average of all the reputation segments. So the parameters of $w_{i: j}^{\text {syn }}$ are calculated as follows.

$$
\begin{aligned}
& b_{i: j}^{s y n}=\frac{1}{\sum_{k \neq i} v_{k, j}^{i}} \sum_{k \neq i} v_{k, j}^{i} b_{k: j}, \\
& d_{i: j}^{s y n}=\frac{1}{\sum_{k \neq i} v_{k, j}^{i}} \sum_{k \neq i} v_{k, j}^{i} d_{k: j}, \\
& u_{i: j}^{s y n}=\frac{1}{\sum_{k \neq i} v_{k, j}^{i}} \sum_{k \neq i} v_{k, j}^{i} u_{k: j} .
\end{aligned}
$$

Clearly, $b_{i: j}^{s y n}, d_{i: j}^{s y n}$ and $u_{i: j}^{s y n}$ satisfy the constraints in Eqn. (1). When node $i$ prepares to update the final reputation value of node $j, w_{i: j}^{s y n}$ represents external knowledge, which will be combined with individual knowledge of node $i, w_{i: j}$. In the subjective logic, for the two opinions, i.e., $w_{i: j}^{s y n}$ and $w_{i: j}$, we can use the defined consensus operation to combine them well and create a final reputation segment $w_{i: j}^{f i n}=w_{i: j} \oplus w_{i: j}^{s y n}$, namely,

$$
\begin{aligned}
& b_{i: j}^{f i n}=\frac{b_{i: j} u_{i: j}^{s y n}+b_{i: j}^{s y n} u_{i: j}}{u_{i: j}^{s y n}+u_{i: j}-u_{i: j}^{s y n} u_{i: j}}, \\
& d_{i: j}^{f i n}=\frac{d_{i: j}^{s y n} u_{i: j}^{s y n}+d_{i: j}^{s y n} u_{i: j}}{u_{i: j}^{s y n}+u_{i: j}-u_{L A: j}^{s y n} u_{i: j}}, \\
& u_{i: j}^{f i n}=\frac{u_{i: j}^{s y n} u_{i: j}}{u_{i: j}^{s y n}+u_{i: j}-u_{L A: j}^{s y n} u_{i: j}} .
\end{aligned}
$$

Based on $w_{i: j}^{f i n}$, the final reputation value of node $j$ is calculated: $r_{i: j}^{f i n}=b_{i: j}^{f i n}+u_{i: j}^{f i n} a_{i: j}^{f i n}$.

\section{Secure and Energy-efficient Communications in SEANET}

To determine the next-hop relay, a node also consider$s$ energy states of it and the neighbor nodes for reliable communications. The node prefers to transmit data packets to closer nodes because of lower energy consumption. In addition, the node transmits the data packets to the relays with sufficient energy. This ensures that the data packets can be routed continuously and finally reach the sink node. Therefore, energy-efficient communications are introduced.

In this paper, we propose an improved Dijkstra algorithm to realize secure and energy-efficient communications when optimizing traffic routing. For node $i$, the cost of being scheduled as a relay in a route path to the sink node is $C_{i}$. Then $C_{i}$ can be periodically updated as follows:

$$
C_{i}=\left\{\begin{array}{l}
\min \left(C_{j}+\omega_{i, j}\right), j \in N_{i} \& i \notin N_{\mathrm{sink}} \\
w_{i, \text { sink }}, i \in N_{\mathrm{sink}}
\end{array}\right.
$$

where $N_{i}$ is the neighbor set of node $i$, and $\omega_{i, j}$ indicates the cost that node $i$ transmits the data packets to node $j$. Consequently, the best relay for node $i$ is acquired as $R_{i}^{*}=\underset{j}{\arg \min }\left(C_{j}+\omega_{i, j}\right)$. To enable secure and energyefficient communications, $\omega_{i, j}$ is defined as follows:

$$
\omega_{i, j}=\frac{\kappa_{1}}{r_{i, j}^{f i n}}+\kappa_{2} \frac{E_{i, j}^{t}}{E_{i}^{r e}}+\kappa_{3} \frac{E_{i, j}^{r}+E_{j, R_{j}^{*}}^{t}}{E_{j}^{r e}} .
$$

$\kappa_{1}, \kappa_{2}$ and $\kappa_{3}$ are three predefined weights and $\kappa_{1}+\kappa_{2}+\kappa_{3}=$ 1. In particular, when node $j$ is the sink node, $\kappa_{1}$ and $\kappa_{3}$ are valued by zero. $E_{i}^{r e}$ and $E_{j}^{r e}$ are residual energy of nodes $i$ and $j$ at the current time, respectively. $E_{i, j}^{t}$ is the energy consumed by node $i$ in case of transmitting $\delta$-bit data packets to node $j$ while $E_{i, j}^{r}$ is the energy consumed by node $j$ resulted by receiving $\delta$-bit data packets from node $i$. According to the proposed energy consumption model in [20], they are calculated by

$$
\begin{aligned}
& E_{i, j}^{t}=\left(\phi_{i}^{t}+\tau d_{i, j}^{2}\right) \delta, \\
& E_{i, j}^{r}=\phi_{j}^{r} \delta .
\end{aligned}
$$

Here, $\phi_{i}^{t}$ and $\phi_{j}^{r}$ is the unit energy cost for nodes $i$ and $j$ to transmit and receive one bit, respectively. $\tau$ is the energy required by an amplifier in free space and $d_{i, j}$ is the distance between node $i$ and node $j$.

To meet the above requirements, the following instructions are written to the data forwarding rule table of node $i$ for achieving secure and energy-efficient communications.

Rule 1: After node $i$ transmits data packets to neighbor node $j$, it updates the individual knowledge about the node, $w_{i: j}$. The reputation segment is uploaded to the SD data controller.

Rule 2: Node $i$ queries, weights and aggregates all the latest reputation segments about node $j$ to form external knowledge $w_{i: j}^{s y n}$. Individual and external knowledge are combined to acquire $w_{i: j}^{\text {fin }}$.

Rule 3: Node $i$ periodically uploads its energy state to the SD energy controller, as proposed in [10]. The period is strictly set by the SD energy controller.

Rule 4: Node $i$ updates its $C_{i}$ and $R_{i}^{*}$ with the neighbor nodes' energy states and reputation values changing. The latest $C_{i}$ and $R_{i}^{*}$ are collected to the SD data controller.

Rule 5: Node $i$ exactly transmits data packets to the nexthop relay according to the determined $R_{i}^{*}$.

\section{Flexible ENERgy Scheduling FOr SEANET}

\section{A. Mode Selection of Bi-directional Energy Transfer}

A mobile charger is scheduled by a charging service provider to visit nodes to provide energy replenishment and collect idle energy. Meanwhile, the mobile charger also acts as a mobile data collector to collect sensory data from nodes. Previous work, e.g., [13] and [16], have studied how to optimize tour planning of the mobile charger. According to periodic scheduling, the mobile charger visits some predefined positions called anchor points in the network. The mobile charger stays at these anchor points with a period of sojourn time so that nearby nodes can transmit their sensory data to it via multi-hop transmissions. Meanwhile, the mobile charge is able to recharge the visited nodes or collect surplus energy via energy downlinks and energy uplinks.

To enable the bi-directional energy transfer, every node should firstly determine whether to work in an energy downlink or energy uplink mode. In an industrial environment, nodes are deployed at static locations and have slightly regular sensing tasks over time. To transmit the sensory data to the sink node, data traffic gives rise to different workloads and energy consumption. In a time period, the detailed energy consumption of nodes can be predictable and estimated via advanced technologies, e.g., in [21]. Besides, energy harvesting characteristics can also be known when available prior 
knowledge is mastered. We consider that the energy harvesting process is a sequence of independent identically distributed (i.i.d.) random variables over time.

Being scheduled, the mobile charger periodically visits each sink node to gather real-time sensory data. The duration of a time period is denoted by $T$. At the beginning of the $t_{t h}$ data gather time period, the residual energy of node $i$ is known as $E_{i}^{t, r e}$. For node $i$, the arrival of harvesting energy follows a Poisson process with the average rate $\lambda_{i}^{t}$. The total amount of consumed energy of node $i$ in the time period is $\mu_{i}^{t}$, whose probability density function is $f\left(\mu_{i}^{t}\right)$. When the residual energy is $E_{i}^{t, r e}$, the probability that node $i$ demands extra energy is

$$
P\left(\mu_{i}^{t}>E_{i}^{t, r e}+\lambda_{i}^{t} T\right)=1-\int_{0}^{E_{i}^{t, r e}+\lambda_{i}^{t} T} f\left(\mu_{i}^{t}\right) d \mu_{i}^{t} .
$$

To ensure normal operations, the above probability should be larger than a presetting threshold value $p_{\text {min }}$. Thus, there is a minimal requirement for $E_{i}^{t, r e}$, as shown by

$$
E_{i}^{t, r e} \geq E_{i}^{t, \min }=F^{-1}\left(1-p_{\min }\right)-\lambda_{i}^{t} T,
$$

where $F^{-1}(\cdot)$ is the inverse function of the cumulative distribution function of $f$.

Clearly, for node $i$, when $E_{i}^{t, r e}$ is smaller than $E_{i}^{t, m i n}$, the node should request energy replenishment. The replenishing amount is equal to $\min \left(E_{i}^{t, m i n}-E_{i}^{t, r e}, B-E_{i}^{t, r e}\right)$, where $B$ indicates the capability of node battery. Otherwise, the node considers uploading its surplus energy via an energy uplink.

\section{B. Nash Bargaining Game for Energy Trading}

In one data gathering period, some busy nodes request for charging service (i.e., energy replenishment) to perform sensing tasks continuously. Besides, energy-rich nodes can sell their surplus energy to the mobile charger. In an energy market, the energy uplinks need to be stimulated by efficient incentive policies. The charging service provider announces an energy purchase price to the nodes according to the dynamic energy demand of intra-network nodes. On the other hand, each energy-rich node makes a decision on the optimal amount of traded energy to maximize its own benefit. The decision is made according to the different energy purchase prices set by the charging service provider and the rationality of the node. Total benefits of an energy trade are allocated to the charging service provider and an energy-rich nodes. This gives rise to a benefit allocation problem in the energy trade. Here, we utilize a Nash bargaining game to formulate and solve this problem.

The charging service provider sets the dynamic energy purchase price. It records the total energy demand in each data gathering period. Then the energy purchase price can be dynamically adjusted to match with current energy demand. In the $t_{t h}$ data gathering period, the energy purchase price for one unit of energy $P_{t}$ is related to the total energy demand in the last data gathering period, $D_{t-1}$. Hence, the charging service provider sets $P_{t}=\gamma_{1}\left(\frac{D_{t-1}}{\gamma_{2}}\right)^{\gamma_{3}}$, where $\gamma_{1}, \gamma_{2}$ and $\gamma_{3}$ are three presetting pricing parameters.

Before an actual energy trade, a negotiation process is executed between the charging service provider and an energyrich node. The mobile charger also represents the charging service provider and can perform multiple negotiation processes at the same time. In the trade, a risk-neutral energy seller and a risk-neutral energy buyer negotiate the benefit allocation. The seller and the buyer in the game obtain allocations according to the Pareto optimality of a Nash bargaining solution or a disagreement after the negotiation. In this paper, we normalize the disagreement points of a negotiation to zero. Thus, the objective function of the negotiation is expressed as follows:

$$
U=\left(\pi_{i}^{t}-0\right)^{\rho_{i}^{t}}\left(\pi_{c, i}^{t}-0\right)^{1-\rho_{i}^{t}} .
$$

The indexes $\rho_{i}^{t}$ and $\left(1-\rho_{i}^{t}\right)$ are defined as the individual negotiation power of node $i$ and the charging service provider, respectively. The individual negotiation power of a node is determined by its maximum amount of energy trading, which is expressed by $\rho_{i}^{t}=1-\exp \left(-\eta\left(E_{i}^{t, r e}-E_{i}^{t, \min }\right)\right)$. $\eta$ is a parameter manipulated by the charging service provider.

Next, we focus on analyzing the detailed benefits of the seller and the buyer in every Nash bargaining process. Let $\pi_{i}^{t}$ and $\pi_{c, i}^{t}$ denote as the benefits of node $i$ and the charging service provider in the $t_{t h}$ data gathering period, respectively. In the $t_{t h}$ time period, if the amount of traded energy is $x_{i}^{t}$, for node $i$, its benefits can be expressed as

$$
\pi_{i}^{t}=n_{i}^{t} P_{t} x_{i}^{t}-\Delta L\left(x_{i}^{t}\right),
$$

where $n_{i}^{t}$ is the share of node $i$ in percentage obtained from the total benefits of the energy trade. Let us denote the potential loss caused by selling $x_{i}^{t}$ amounts of energy by $\Delta L\left(x_{i}^{t}\right)$, which can be expressed as $\Delta L\left(x_{i}^{j}\right)=\frac{l_{i}}{\rho_{i}^{t}}\left(x_{i}^{t}\right)^{2}$. Here, $l_{i}$ is defined as the preferred loss factor. The negotiation power influences the potential loss as more surplus energy will reduce certain threats causing by selling $x_{i}^{t}$ amount of energy. For the charging service provider, its benefits are calculated as

$$
\pi_{c, i}^{t}=\left(1-n_{i}^{t}\right) P_{t} x_{i}^{t}-c_{i} x_{i}^{t},
$$

where $\left(1-n_{i}^{t}\right)$ is the share of the charging service provider in percentage, and $c_{i}$ is the average fixed cost for transferring one unit of energy. The charging service provider undertakes the energy transfer costs in the energy uplink.

To maximize the utility of the negotiation, $\ln U$ is differentiated with respect to $n_{i}^{t}$.

$$
\begin{aligned}
& \left.\frac{\partial \ln U}{\partial n_{i}^{t}}=\frac{P_{t} x_{i}^{t} \rho_{i}^{t}}{n_{i}^{t} P_{t} x_{i}^{t}-\left(l_{i} / \rho_{i}^{t}\right) x_{i}^{t^{2}}}-\frac{P_{t} x_{i}^{t}\left(1-\rho_{i}^{t}\right)}{\left(1-n_{i}^{t}\right) P_{t} x_{i}^{t}-c_{i} x_{i}^{t}}\right) \\
& \frac{\partial \ln ^{2} U}{\partial n_{i}^{t 2}}=-\frac{P_{t}^{2}}{\left(n_{i}^{j} P_{t} x_{i}^{t}-P_{t}^{2} t_{i}^{t^{2}}\left(l_{i}^{t} / \rho_{i}\right) x_{i}^{t 2}\right)^{2}}-\frac{P_{t}^{2} x_{i}^{2}\left(1-\rho_{i}^{t}\right)}{\left(\left(1-n_{i}^{t}\right) P_{t} x_{i}^{t}-c_{i} x_{i}^{t}\right)^{2}}<0 .
\end{aligned}
$$

Clearly, the objective function of the negotiation is concave, which indicates that the maximal value of this function exists. Therefore, using the first-order optimality condition $\partial \ln \mathrm{U} / \partial n_{i}^{t}=0$, we obtain the best response of the negotiation in terms of node $i$, as follows,

$$
n_{i}^{t *}=\frac{\left(1 / \rho_{i}^{t}-1\right) l_{i}\left(x_{i}^{t}\right)^{2}+\rho_{i}^{t} P_{t} x_{i}^{t}-\rho_{i}^{t} c_{i} x_{i}^{t}}{P_{t} x_{i}^{t}} .
$$

Equation (20) shows the final share of node $i$ in percentage obtained from the energy trade. The equation encourages fairness of energy trading as the charging service provider actually gives the payments $\left(P_{t} x_{i}^{t}\right)$ to node $i$ and also undertakes the energy transfer costs $\left(c_{i} x_{i}^{t}\right)$. So the final share of node $i$ in percentage decreases with increasing payments and energy 
transfer costs. On the other hand, the charging service provider aims to stimulate energy-rich nodes to upload more energy. Then the nodes can obtain higher shares when they are able to upload more their surplus energy. Namely, $n_{i}^{t *}$ is increased with higher individual negotiation power, $\rho_{i}^{t}$.

By substituting $n_{i}^{t *}$ into $\pi_{i}^{t}$, we can see that the benefit function of node $i$ can be converted into an optimal function in terms of $x_{i}^{t}$ as follows:

$$
\pi_{i}^{t *}=-l_{i} x_{i}^{t 2}+\rho_{i}^{t}\left(P_{t}-c_{i}\right) x_{i}^{t} .
$$

We take the first and second derivatives of $\pi_{i}^{j *}$ with respect to $x_{i}^{j}$,

$$
\begin{aligned}
& \frac{\partial \pi_{i}^{t *}}{\partial x_{i}^{t}}=-2 l_{i} x_{i}^{t}+\rho_{i}^{t}\left(P_{t}-c_{i}\right) \\
& \frac{\partial^{2} \pi_{i}^{t *}}{\partial x_{i}^{t 2}}=-2 l_{i}<0 .
\end{aligned}
$$

We find that the optimal function is also concave. By using $\partial \pi_{i}^{t *} / \partial x_{i}^{t}=0$, the optimal amount of traded energy for node $i$ takes the form $x_{i}^{t *}$ :

$$
x_{i}^{t *}=\frac{\rho_{i}^{t}\left(P_{t}-c_{i}\right)}{2 l_{i}} .
$$

Theorem 1 : With the solution $n_{i}^{t *}$ and $x_{i}^{t *}$, Nash equilibrium can be achieved in the Nash bargaining game.

Proof: As it is stated above, within $n_{i}^{t *}$ and $x_{i}^{t *}$, the node can acquire the best utility $\pi_{i}^{t *}$. For the charger service provide, $\pi_{c, i}^{t}$ is differentiated with respect to $x_{i}^{t}$ as follows:

$$
\begin{aligned}
& \frac{\partial \pi_{c, i}^{t}}{\partial x_{i}^{t}}=P_{t}-\frac{\partial n_{i}^{t}}{\partial x_{i}^{t}} P_{t} x_{i}^{t}-n_{i}^{t} P_{t}-c_{i} \\
& \frac{\partial^{2} \pi_{c, i}^{t}}{\partial x_{i}^{t 2}}=-\frac{\partial^{2} n_{i}^{t}}{\partial x_{i}^{t 2}} P_{t} x_{i}^{t}-2 \frac{\partial n_{i}^{t}}{\partial x_{i}^{t}} P_{t} .
\end{aligned}
$$

According to Eqn. (18), we easily get that

$$
\begin{aligned}
& \frac{\partial n_{i}^{t *}}{\partial x_{i}^{t}}=\left(\frac{1}{\rho_{i}^{t}}-1\right) \frac{l_{i}}{P_{t}}>0, \\
& \frac{\partial^{2} n_{i}^{j *}}{\partial x_{i}^{j 2}}=0 .
\end{aligned}
$$

By substituting Eqns. (18), (21) and (23) into Eqn. (22), we find that $\partial \pi_{c, i}^{t} / \partial x_{i}^{t}=0$ and $\partial^{2} \pi_{c, i}^{t} / \partial x_{i}^{t 2}<0$ are satisfied. This means that the maximal utility of the charger service provider is also obtained under the condition of $n_{i}^{t *}$ and $x_{i}^{t *}$. In conclusion, both the node and the charger service provider are fully satisfied because that the decisions (i.e., $n_{i}^{t *}$ and $x_{i}^{t *}$ ) make that their utilities have been maximized simultaneously. So they have agreed upon the solution and also have no motivation to change their decisions and take other actions. Finally, Nash equilibrium is achieved in the Nash bargaining game. Theorem 1 can be proved.

\section{NUMERICAL RESULTS}

In this section, we evaluate the performance of proposed SEANET through extensive simulations. We consider an SEANET with coverage size $80 \mathrm{~m} \times 80 \mathrm{~m}$, and consists of 4 monitoring areas. Every monitoring area has 30 randomly deployed nodes, whose communication range is $10 \mathrm{~m}$ and capacity of each node battery is $1 \mathrm{~J}$. During communications, energy consumption parameters are based on that in [20]. The mobile charger with sufficient energy travels around all the monitoring areas to gather data every 30 minutes [16]. During
TABLE I: Parameter Setting in the Simulation

\begin{tabular}{l|c}
\hline Parameter & Values \\
\hline Network size & $80 \mathrm{~m} \times 80 \mathrm{~m}$ \\
\hline The number of monitoring areas & 4 \\
\hline Quality of link $q$ & $U \sim[0.7,0.95]$ \\
\hline $\begin{array}{l}\text { Parameters of formulation the } \\
\text { freshness } \alpha_{1} \text { and } \alpha_{2}\end{array}$ & $10,1.2$ \\
\hline $\begin{array}{l}\text { Weights of a reputation segment } \\
\beta_{1}, \beta_{2} \text { and } \beta_{3}\end{array}$ & $0.3,0.3,0.4$ \\
\hline $\begin{array}{l}\text { Energy consumption parameters } \\
\phi^{t}, \phi^{r} \text { and } \tau\end{array}$ & $50 \mathrm{~nJ} / \mathrm{bit}, 50 \mathrm{~nJ} / \mathrm{bit}, 100 \mathrm{pJ} / \mathrm{bit} / \mathrm{m}^{2}$ \\
\hline Weights of traffic control $\kappa_{1}, \kappa_{2}$ & $0.5,0.3,0.2$ \\
and $\kappa_{3}$ & {$[0.1,0.3] \mathrm{mJ} / \mathrm{s}$} \\
\hline Energy harvesting process $\lambda$ & $3,11,-1.1$ \\
\hline Pricing parameters $\gamma_{1}, \gamma_{2}$ and $\gamma_{3}$ & $U \sim[1,3]$ \\
\hline Loss factor $l$ & $U \sim[0,1]$ \\
\hline Average energy transfer cost $c$ & \\
\hline
\end{tabular}

an energy trading, the parameter for formulating individual negotiation power $\eta$ is set as 1.35. More simulation parameters can be found in Table 1 .

\section{A. Performances Evaluation of Data Communications}

In SEANET, secure and energy-efficient communications are introduced for reliable data traffic. In particularly, we use the multi-weighted subjective logic (MWSL) to formulate reputation values of nodes. A widely adopted model of reputation computation using the subjective logic is a linear function proposed in [22]. Compared with the traditional subjective logic (TSL), our approach takes weighting operation for diverse reputation segments into consideration. The reputation segments with greater qualities are distinguished and emphasized for reputation aggregation, achieving highaccuracy reputation computation.

We consider a scenario with 5 misbehaving nodes randomly deployed in a monitoring area. Data arrival process of each node follows a Poisson process and average arrival rate ranges from 2 to $10 \mathrm{~kb} / \mathrm{s}$. The probability of dropping relayed data packets is used to formulate the behavior of nodes. We set the observing time as 10 minutes. Initially, the misbehaving nodes pretend to act cooperatively. The probability of normally relaying data packets reaches to $90 \%$. After a time period of time, e.g., 3 minutes, the misbehaving nodes show the misbehavior and the probability is dropped to $10 \%$.

To realize the misbehavior, their neighbors update their reputation values. The reputation values of 5 misbehaving $\mathrm{n}$ odes are updated by two approaches, i.e., traditional subjective logic and multi-weighted subjective logic. Fig. 4 shows that the reputation values calculated by MWSL are lower than those calculated by the TSL. By weighting, the reputation segments, that are up-to-date and have more prior knowledge for the misbehaving nodes, are referenced with emphasis. So the misbehavior is detected more clearly. From the figure, after the same observing time, the average reputation value of the misbehaving nodes updated by MWSL is about 29\% lower than that updated by TSL. This means that our approach is much sensitive for the misbehavior and can distinguish the misbehaving nodes more timely. 


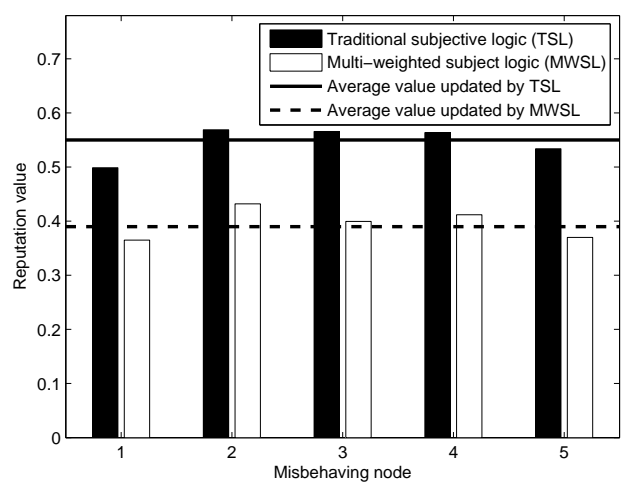

Fig. 4: The comparison of reputation values of misbehaving nodes updated by two reputation computation methods.

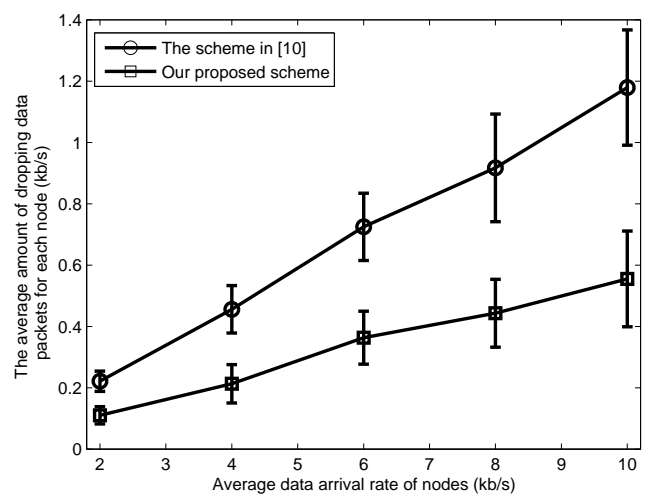

Fig. 5: The comparison of the average amount of dropping data packets between the existing scheme and our scheme.

As a result, the misbehaving nodes are detected and not selected as relays when routing traffic. The existing scheme in [10] also utilizes SDN controllers to facilitate energyefficient communications. But the work neglects the existence of misbehaving nodes. As shown in Fig. 5, we observe the amount of packet loss within 100 seconds and the observation is repeated 100 times. Clearly, with the higher data arrival rate, the total amount of dropping data packets caused by the misbehaving nodes is increased. When the average data arrival rate of nodes is $8 \mathrm{~kb} / \mathrm{s}$, the average amount of dropping data packets is only $0.44 \mathrm{~kb} / \mathrm{s}$ for each node in our scheme. But that is up to $0.92 \mathrm{~kb} / \mathrm{s}$ in the scheme of [10]. Thus, our work achieves more than $52 \%$ decline rate for lessening the amount of dropping data packets after considering secure communications.

\section{B. Performances Evaluation of Energy Scheduling}

In SEANET, energy-deficient nodes can replenish energy through two ways: i) energy replenishment from the mobile charger, and ii) energy scheduling among nodes. For supporting energy scheduling, the mobile charger transfers the surplus energy from energy-rich nodes to energy-deficient nodes. We consider that there are two busy monitoring areas with higher energy consumption and two idle monitoring areas with lower

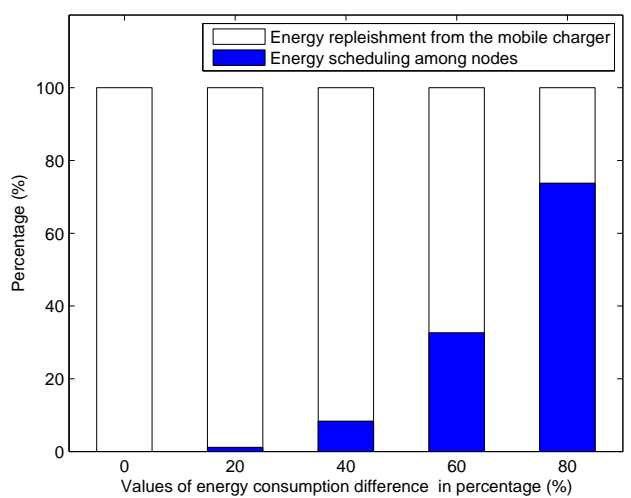

Fig. 6: The comparison of energy from external replenishment and internal scheduling in percentage.

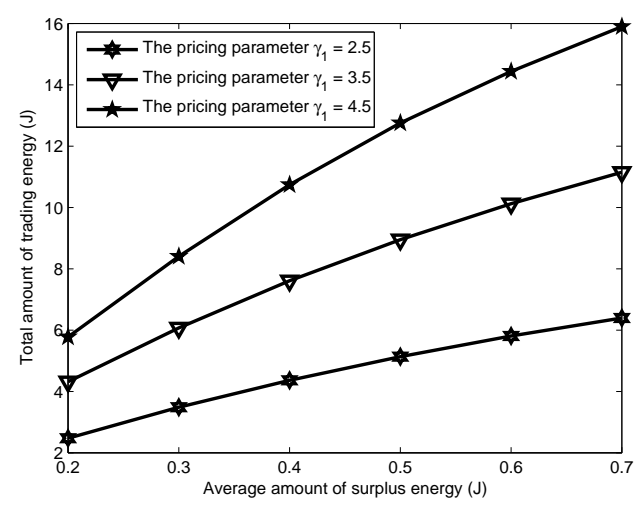

Fig. 7: The comparison of the total amount of trading energy between the nodes and mobile charger.

energy consumption. The amount of consumed energy range within a data gather time period ranges from $0.3 \mathrm{~J}$ and $1.8 \mathrm{~J}$, following a normal distribution. The threshold value $p_{\min }$ is 0.1 and the amount of surplus energy from a idles node can range from $0.2 \mathrm{~J}$ to $0.7 \mathrm{~J}$ in the simulation.

Fig. 6 shows the percentage comparison between these two energy replenishment ways. There are five kinds of energy consumption differences in percentage between idle monitoring areas and busy monitoring areas. When the value of energy consumption difference increases, the percentage of first way is decreasing while the percentage of second ways is increasing. As all nodes are busy, there exists little energy consumption difference, resulting in inexistence of energy uplinks. As a result, energy-deficient nodes replenish energy from the mobile charger. However, when the value of energy consumption difference is bigger (e.g., the value is $80 \%$ in percentage), the percentage of energy scheduling among nodes is about $180 \%$ higher than that of the first way. Surplus energy from the energy-rich nodes encourages energy uplinks. Then more energy-deficient nodes can benefit from this flexible energy scheduling. Thus, SEANET is beneficial to decrease external energy replenishment to the network, especially under the condition of obvious energy consumption difference.

Fig. 7 shows the performance comparison of total amount 


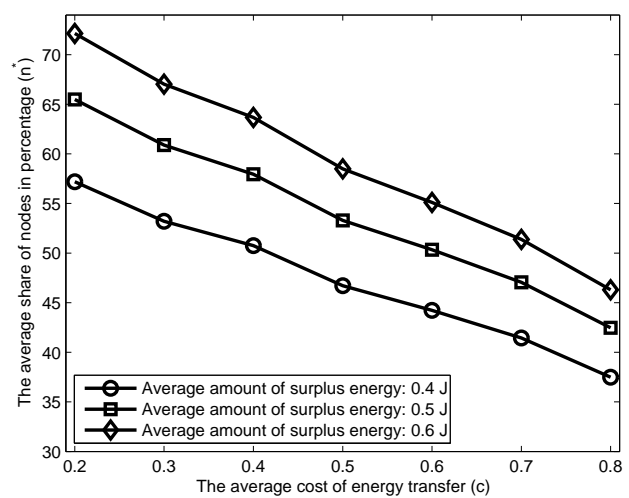

(a) Performance comparison of average share of nodes in terms of average amount of surplus energy and energy transfer cost $(c)$.

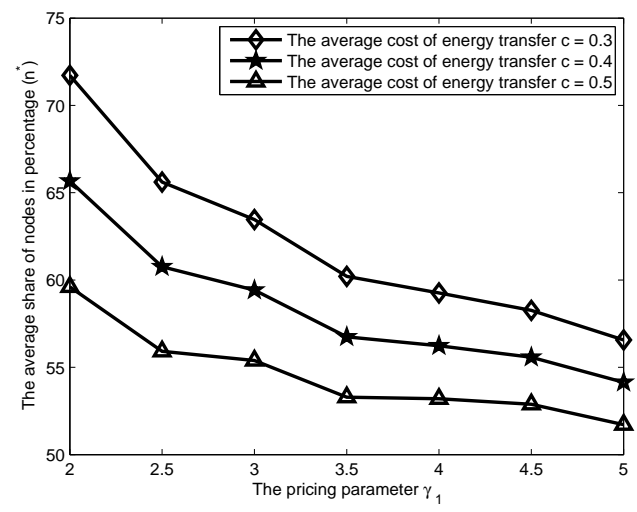

(b) Performance comparison of average share of nodes in terms of pricing parameter $\gamma_{1}$ and energy transfer $\operatorname{cost}(c)$.

Fig. 8: The comparison of average share of nodes in the energy trading.

of trading energy with respect to different average amounts of surplus energy and different pricing parameters $\gamma_{1}$. Due to different amounts of surplus energy, the total amount of trading energy is changing. Higher amounts of surplus energy brings more trading energy. Moreover, when the pricing parameter $\gamma_{1}$ is higher, energy trades are encouraged and the nodes would like to trade more energy.

Fig. 8(a) and Fig. 8(b) show the performance comparison of benefit shares (i.e., $n^{*}$ ) with respect to different average amounts of surplus energy from the nodes, energy transfer costs $(c)$ and pricing parameters $\left(\gamma_{1}\right)$. For nodes, increasing amount of surplus energy brings more trading energy, leading to higher negotiation power in the energy trades. Finally, the nodes can obtain higher average share in the energy trades, as shown in Fig. 8(a). But the share of the nodes decreases when the energy transfer cost and pricing parameter are increasing. The charging service provider undertakes higher energy transfer costs and payments, the share of the charging service provider should have been increased for fairness of energy trading. Then the average share of the nodes decreases. For example, in Fig. 8(a), when the average amount of surplus energy is $0.6 \mathrm{~J}$, the share of the charging service provider reaches to about $59 \%$ due to the higher value of $c, 0.7$. Similar results can be found in Fig. 8(b).

\section{CONCLUSION}

Energy harvesting IoT is emerged when energy harvesting technology and energy replenishment from the mobile charger are proposed to prolong network lifetime. Bi-directional energy flows are formed as energy-rich nodes are stimulated to upload surplus energy to the mobile charger. But energy flows couple with data flows, and new problems on controlling these flows should be addressed. In this paper, we propose SEANET wherein the data plane, energy plane and control plane are decoupled for centralized control of the flows. In SEANET, we use the multi-weighted subjective logic to compute reputation values of nodes. Traffic is routed among the nodes with higher reputation values and sufficient energy for reliable communications. Besides, to support flexible energy scheduling, a Nash bargaining game is formulated to solve the benefit allocation problem for feasible energy trading in SEANET. Numerical results indicate that SEANET improves data traffic by reducing packet loss, optimizes energy utilization and saves energy.

For future work, it is an interesting direction to extend the core idea of SEANET to other scenarios that energy flows coexist with data flows. For example, SEANET could be integrated into existing offline mobile social networks wherein socially-close users can exchange content files during frequent encounters via the Device-to-Device technology [23], [24]. Two mobile users can also share energy with each other if necessary, and bi-directional energy flows arise up. But new problems are emerged, because complex social interactions among mobile users should be considered when scheduling energy flows and data flows in dynamic environments.

\section{REFERENCES}

[1] A. Al-Fuqaha et al., "Internet of things: A survey on enabling technologies, protocols, and applications," IEEE Communications Surveys Tutorials, vol. 17, pp. 2347-2376, Fourthquarter 2015.

[2] S. Li, L. D. Xu, and S. Zhao, "The internet of things: a survey," Information Systems Frontiers, vol. 17, pp. 243-259, Apr. 2015.

[3] X. Wang et al., "Tag-assisted social-aware opportunistic device-to-device sharing for traffic offloading in mobile social networks," IEEE Wirel. Comm., vol. 23, pp. 60-67, August 2016.

[4] P. Kamalinejad et al., "Wireless energy harvesting for the internet of things," IEEE Comm. Magazine, vol. 53, pp. 102-108, June 2015.

[5] H. Zhang et al., "Energy efficient user association and power allocation in millimeter-wave-based ultra dense networks with energy harvesting base stations," IEEE J. Sel. Area. Comm., vol. 35, pp. 1936-1947, Sept. 2017.

[6] Y. Xiao et al., "Dynamic energy trading for energy harvesting communication networks: A stochastic energy trading game," IEEE J. Sel. Area. Comm., vol. 33, pp. 2718-2734, Dec. 2015.

[7] A. L. Valdivieso Caraguay et al., "Sdn: Evolution and opportunities in the development iot applications," International Journal of Distributed Sensor Networks, vol. 10, no. 5, pp. 735-742, 2014.

[8] D. Kreutzand et al., "Software-defined networking: A comprehensive survey," Proceedings of the IEEE, vol. 103, pp. 14-76, Jan. 2015.

[9] M. Ojo, D. Adami, and S. Giordano, "A sdn-iot architecture with nfv implementation," IEEE Globecom Workshops, pp. 1-6, Dec. 2016.

[10] J. Wang et al., "A software defined network routing in wireless multihop network," Journal of Network and Computer Applications, vol. 85, no. Supplement C, pp. 76 - 83, 2017.

[11] C. Li et al., "Securing sdn infrastructure of iot-fog networks from mitm attacks," IEEE Internet of Things Journal, vol. 4, pp. 1156-1164, Oct. 2017.

[12] D. Zeng et al., "Energy minimization in multi-task software-defined sensor networks," IEEE Trans. on Comput., vol. 64, pp. 3128-3139, Nov. 2015. 
[13] M. Zhao et al., "A framework of joint mobile energy replenishment and data gathering in wireless rechargeable sensor net- works," IEEE Trans. on Mobil. Comput., vol. 13, pp. 2689-2705, Dec. 2014.

[14] X. Huang et al., "Software defined energy harvesting networking for $5 \mathrm{~g}$ green communications," IEEE Wirel. Comm., vol. 24, no. 4, pp. 38-45, 2017.

[15] Open Networking Foundation (ONF), 2014. [Online]. Available: https: //www.opennetworking.org/.

[16] S. Guo, C. Wang, and Y. Yang, "Joint mobile data gathering and energy provisioning in wireless rechargeable sensor networks," IEEE Trans. on Mobil. Comput., vol. 13, pp. 2836-2852, Dec. 2014.

[17] A. Josang, "A logic for uncertain probabilities," International Journal of Uncertainty, Fuzziness and Knowledge-Based Systems, vol. 9, no. 3, pp. 279-311, 2001.

[18] M. Salehi et al., "A trust-based security approach in hierarchical wireless sensor networks," Ad Hoc Networks, vol. 7, no. 6, pp. 58-67, 2017.

[19] G. Liu et al., "Assessment of multi-hop interpersonal trust in social networks by three-valued subjective logic," IEEE INFOCOM, pp. 16981706, April 2014.

[20] W. R. Heinzelman et al., "Energy-efficient communication protocol for wireless microsensor networks," Proceedings of the 33rd Annual Hawaii International Conference on System Sciences, pp. 1-10, Jan. 2000.

[21] I. Mller et al., "Algorithm for estimation of energy consumption of industrial wireless sensor networks nodes," IEEE International Instrumentation and Measurement Technology Conference Proceedings, pp. 440-444, May 2014.

[22] S. Zhong, J. Chen, and Y. R. Yang, "Sprite: a simple, cheat-proof, creditbased system for mobile ad-hoc networks," INFOCOM, March 2003.

[23] X. Wang et al., "D2D big data: Content deliveries over wireless deviceto-device sharing in realistic large scale mobile networks," IEEE Wirel. Comm., vol. 25, pp. 1-10, Feb. 2018.

[24] S. Wang et al., "Large scale measurement and analytics on social groups of device-to-device sharing in mobile social networks," Mobile Networks and Applications, Springer, pp. 1-13, Sept. 2017.

\section{BIOGRAPHIES}

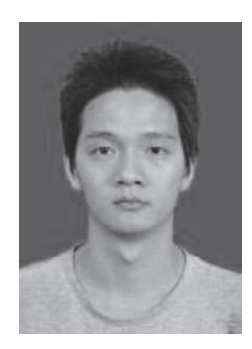

Xumin Huang is now pursuing his Ph.D. degree in Guangdong University of Technology, China. His research interests mainly focus on network performance analysis, simulation and enhancement in wireless communications and networking.

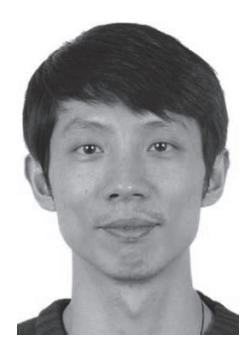

Rong Yu (M'08) received his Ph.D. degree from Tsinghua University, China, in 2007. After that, he worked in the School of Electronic and Information Engineering of South China University of Technology. In 2010, he joined the Institute of Intelligent Information Processing at Guangdong University of Technology, where he is now a full professor. His research interests include wireless networking and mobile computing in featured environments such as Edge Cloud, Connected Vehicles, Smart Grid, and Internet of Things. He is the co-inventor of over 30 patents and author or co-author of over 100 international journal and conference papers. He was the member of home networking standard committee in China, where he led the standardization work of three standards.

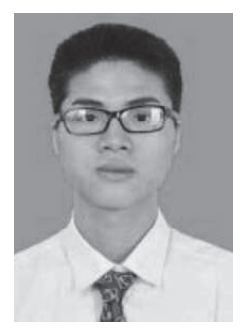

Jiawen Kang received the M.S. degree from the Guangdong University of Technology, China, in 2015. He is now pursuing his Ph.D. degree in Guangdong University of Technology, China. His research interests mainly focus on resource management, security and privacy protection in wireless communications and networking.

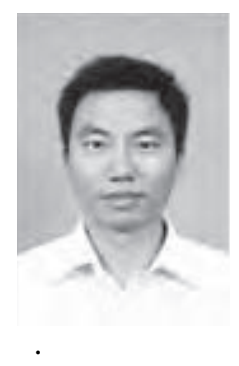

Zhuoquan Xia received his Ph.D.degree from Central South University in 2012. He is presently a Professor of Changsha University of Science and Technology. His main research interests include cyber security of the smart grid and IoT, wireless networks.

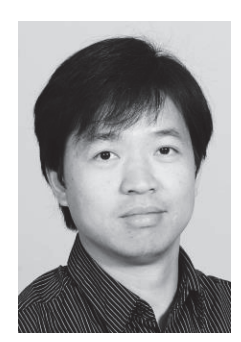

Yan Zhang (SM'10) is currently full Professor at the Department of Informatics, University of Oslo, Norway. He received a Ph.D. degree in School of Electrical \& Electronics Engineering, Nanyang Technological University, Singapore. He is an Associate Technical Editor of IEEE Communications Magazine, an Editor of IEEE Network Magazine, IEEE Transactions on Green Communications and Networking, IEEE Communications Surveys \& Tutorials, IEEE Vehicular Technology Magazine, and the IEEE Internet of Things Journal, and an Associate Editor of IEEE Access. He has served as Chair for a number of conferences, including IEEE GLOBECOM 2017, IEEE VTC-Spring 2017, IEEE PIMRC 2016, IEEE CloudCom 2016, IEEE ICCC 2016, IEEE CCNC 2016, IEEE SmartGridComm 2015, and IEEE CloudCom 2015. He has served as a TPC member for numerous international conference including IEEE INFOCOM, IEEE ICC, IEEE GLOBECOM, and IEEE WCNC. His current research interests include next-generation wireless networks leading to $5 \mathrm{G}$, and green and secure cyber-physical systems (e.g., smart grid, healthcare, and transport). He is an IEEE Vehicular Technology Society Distinguished Lecturer. He is also a Senior Member of IEEE ComSoc, IEEE CS, IEEE PES, and IEEE VT Society. He is a Fellow of IET. 